\title{
Diffusion-weighted MRI of metastatic liver lesions: is there a difference between hypervascular and hypovascular metastases?
}

\author{
Christine Schmid-Tannwald ${ }^{1,2}$, Stephen Thomas', \\ Marko K Ivancevic ${ }^{1,3}$, Farid Dahi', Carsten Rist ${ }^{2}$, lla Sethi' and \\ Aytekin Oto'
}

\begin{abstract}
Background: Different perfusion characteristics and histopathologic features of liver metastasis may potentially lead to different diffusion-weighted magnetic resonance imaging (DW-MRI) characteristics which can affect the performance of DW-MRI in their diagnosis.

Purpose: To compare ADC values of hypervascular and hypovascular metastases and the added value of DW-MRI to T2-weighted (T2-w) images in their detection.

Material and Methods: In this retrospective study, 46 patients (2I with hypervascular, 25 with hypovascular liver metastases) who had undergone abdominal MRI were included. Two independent observers first reviewed T2-w images only and then T2-w + DW-MR images and recorded number of metastases in each session. Lesion detection rate was compared using McNemar test. ADC of metastases in each patient was measured and compared between hypo- and hypervascular lesions using t-test.

Results: A total of 153 hypervascular and 187 hypovascular metastases were detected at consensus review. Two observers detected significantly more hypervascular metastases on T2-w + DW-MR image review session compared to T2-w image only review session (reader I: I48 [96.7\%] vs. I29 [84.3\%], $P=0.002$; reader $2: 125$ [8I.9\%] vs. II3 [73.8\%], $P=0.004)$. Detection rate of hypovascular metastases was similar between two sessions for both observers (reader I: 180 [96.2\%] vs. I84 [98.4\%]; reader 2: 176 [94.I\%] vs. I80 [96.2\%], P> 0.05). The mean ADC value of hypervascular metastases was significantly lower than mean ADC value of hypovascular metastases $\left(1.23+/-0.31 \times 10^{-3} \mathrm{~mm}^{2} / \mathrm{s}\right.$ vs. $\left.1.49+/-0.19 \times 10^{-3} \mathrm{~mm}^{2} / \mathrm{s}\right)(P=0.001)$.

Conclusion: Liver metastases are not a homogenous group of lesions with uniform DW-MRI features. Hypervascular metastases demonstrate significantly lower ADC values compared to hypovascular metastases. DW-MRI improved detection of hypervascular metastases compared to T2-w images alone and is a useful adjunct to T2-w images for their detection.
\end{abstract}

\section{Keywords}

Diffusion-weighted MRI, hypovascular and hypovascular liver metastases, ADC measurements

Date received: 28 October 20I2; accepted: 24 July 2013

\section{Introduction}

Liver metastases are the most common hepatic malignant masses. Based on their blood supply, they may be categorized as hypervascular and hypovascular.

Hypervascular metastases have predominantly hepatic arterial blood supply and appear hyperintense in

\footnotetext{
'The University of Chicago, Department of Radiology, Chicago, IL, USA ${ }^{2}$ Ludwig-Maximilians-University Hospital Munich, Institute for Clinical Radiology, Munich, Germany

${ }^{3}$ Philips Healthcare, MR Clinical Science, Cleveland, OH, USA
}

\section{Corresponding author:}

Christine Schmid-Tannwald, Ludwig-Maximilians-University Hospital Munich, Institute for Clinical Radiology, Marchioninistr. 15, 801377

Munich, Germany.

Email: christine.schmid-tannwald@med.uni-muenchen.de 
the arterial contrast-enhanced magnetic resonance imaging (MRI) whereas they show wash-out on delayed images $(1,2)$. They are usually from neuroendocrine tumors, renal cell carcinoma, subtypes of breast cancer, melanoma, leiomyosarcoma, gastrointestinal stromal tumors, medullary thyroid cancer, and choriocarcinoma (2-4). On contrast-enhanced MRI, hypovascular metastases demonstrate delayed and decreased enhancement compared to the normal liver parenchyma with occasionally rim enhancement $(1,5,6)$. Their primary tumors include gastrointestinal tract, lung, pelvis, pancreas, and prostate cancer (2-4).

MRI is considered to be one of the best and common imaging modality for the detection and accurate characterization of focal liver lesions $(7,8)$. Gadolinium (Gd)-enhanced T1-weighted (T1-w) images are the crucial part of liver MRI protocol and can improve the detection, anatomic depiction and characterization of liver lesions (2-4). However, the use of gadolinium may be contraindicated in patients with severe renal failure due to the risk of nephrogenic systemic fibrosis (NSF) (9). Recently, diffusion-weighted MRI (DW-MRI) has been shown to improve detection of liver metastases and significant differences were found between ADC measurements from between malignant and benign liver lesions (10). DW-MRI has been suggested as a valuable adjunct to $\mathrm{T} 2$-weighted $(\mathrm{T} 2-\mathrm{w})$ images in detection of liver metastases $(11,12)$. However, in all of these studies, metastases were considered as a single homogenous group of lesions and DW-MRI features of hypovascular and hypervascular metastases have not been separately described. Different perfusion characteristics and histopathologic features of these lesions may potentially lead to different DW-MRI characteristics which can affect the performance of DW-MRI in their diagnosis.

The purpose of this study was to compare ADC values of hypervascular and hypovascular metastases and the added value of DW-MRI to T2-w images in their detection.

\section{Material and Methods}

This retrospective HIPPA compliant study was approved by our Institutional review board and waiver of informed consent was obtained. Retrospective search in the Radiology database identified 52 consecutive patients with confirmed liver metastases and underwent Gd-enhanced liver MRI between January 2008 and March 2010. Forty-six patients (mean age, 62.0 years; range, $30-85$ years; 23 women, 23 men) were included. Six patients who did not undergo DWMRI were excluded.

The diagnosis of metastases was confirmed in 15 patients with biopsy of a representative liver lesion.
In six patients, diagnosis was based on the combination of positive PET-CT and follow-up imaging. The diagnosis of metastases was confirmed in 25 patients by follow-up imaging that demonstrated interval progression and/or typical enhancement $(1,2,5,6)$.

One radiologist (observer 1: AO with 12 years of experience in abdominal MRI) reviewed clinical records and gadolinium-enhanced MRI and divided the patients into two groups. Imaging criteria for hypervascular metastases include arterial contrast enhancement with wash-out on delayed images. For hypovascular metastases imaging features were defined as delayed enhancement compared to the normal liver parenchyma with occasionally rim enhancement. In addition clinical records and pathologic findings were used to evaluate, if the primary tumor of each patient tend to cause hypervascular metastases according to the literature (2-4).

Group 1 consisted of patients ( $n=21$; mean age, 59.0 years; range, 30-77 years; 9 women, 12 men) with hypervascular liver metastases (primary malignancy: neuroendocrine tumor $[n=11]$, medullary thyroid cancer $[n=4]$, breast cancer $[n=3]$, renal cell carcinoma $[n=3])$. Group 2 consisted of patients $(n=25$; mean age, 64.5 years; range, 45-85 years; 14 women, 11 men) with hypovascular liver metastases (primary malignancy: pancreatic carcinoma $[n=10]$, colorectal cancer $[n=9]$, ovarian cancer $[n=2]$, esophageal carcinoma $[n=1]$, gastric cancer $[n=1]$, lung cancer $[n=1]$, and breast cancer $[n=1])$.

\section{$M R I$}

Forty-six patients underwent MRI on a $1.5 \mathrm{~T}$ clinical MR systems (Signa HdX, GE Healthcare, Waukesha, WI, USA $[n=40]$; Achieva, Philips Healthcare, Best, The Netherlands $[n=6])$ using 8- (GE) and 16- (Philips) channel phased array body coils.

The imaging protocol consisted of axial T2-w fast spin echo sequence with fat suppression, axial DW single shot EPI sequence with fat saturation and respiratory gating $\left(b=0,800 \mathrm{~s} / \mathrm{mm}^{2}, 5-7 \mathrm{~mm}\right.$ section thickness), and axial contrast-enhanced breath-hold 3D T1-w fat-suppressed gradient echo sequence with intravenous contrast injection (gadodiamide, $0.1 \mathrm{mmol} / \mathrm{kg}$ of body weight $[n=25]$, gadobenate dimeglumine, $0.05 \mathrm{mmol} / \mathrm{kg}$ of body weight $[n=21]$ ). Detailed acquisition protocols were as follows:

GE Healthcare Unit Protocol. An array spatial sensitivity encoding technique (ASSET) (parallel imaging) factor of two was used in all sequences. T2-w FSE sequence with fat suppression parameters were as follows: repetition time/echo time (TR/TE), $8000 / 80 \mathrm{~ms}$; matrix, $256 \times 256$; bandwidth [bw], $62.5 \mathrm{kHz}$; slice/gap, 
7/1 mm; number of excitations, 2; and field of view [FOV], $350 \mathrm{~mm}$. DW imaging parameters were as follows: TR/TE, $\quad 3000-10,000 / 70-80 \mathrm{~ms}$; matrix: $128 \times 128-224 ; b$ factor $=0$ and $800 \mathrm{~s} / \mathrm{mm}^{2}$; bw, $64 \mathrm{kHz}$; number of signals acquired, 4 ; slice/gap, $5 / 2 \mathrm{~mm}$; acquisition time, 2-4 min; and FOV, 320-500 mm. Dynamic contrast-enhanced MRI parameters were as follows: TR/ TE, 4.2-4.6/2-2.3 ms; matrix, $320 \times 192$; flip angle, $10^{\circ}$; bw, $62.5 \mathrm{kHz}$; number of signal acquired, 1; slice thickness/interval, 5/2.5; and FOV, 300-380 mm. For dynamic contrast-enhanced MRI, a fix delay of $25 \mathrm{~s}$ was used for arterial phase images. Arterial and portal venous phase sequences were started 25 and $70 \mathrm{~s}$ after administration of IV contrast. The third phase was obtained immediately after the portal venous phase imaging. Each phase was obtained during separate breathholds. Injection rate: $2 \mathrm{~mL} / \mathrm{s}$.

Philips Healthcare Unit Protocol. An effective sensitivity encoding (SENSE) (parallel imaging) factor of two was used in all sequences. T2-w turbo spin echo with fat suppression imaging parameters were as follows: TR/TE, $1800 / 80 \mathrm{~ms}$; matrix, $332 \times 285$, bw, $579 \mathrm{~Hz} /$ pixel; slice/ gap, $7 / 0 \mathrm{~mm}$; and FOV, $350 \mathrm{~mm}$. DW imaging parameters were as follows: TR/TE, $3578 / 67 \mathrm{~ms} ; b$-factors, 0 and $800 \mathrm{~s} / \mathrm{mm}^{2}$; matrix, $200 \times 128$; bw, $13.4 \mathrm{~Hz} /$ pixel; number of signals acquired, 4 ; slice/gap, $7 / 0 \mathrm{~mm}$; acquisition time (excluding respiratory triggering), 3:27; and FOV, $400 \mathrm{~mm}$. Dynamic contrast-enhanced MRI parameters were as follows: TR/TE, $7.8 / 2.5 \mathrm{~ms}$; matrix, $200 \times 169$; flip angle, $10^{\circ}$; bw, $432 \mathrm{~Hz} /$ pixel; number of signals acquired, 1 ; slice thickness/interval, $4 / 2 \mathrm{~mm}$; breath-hold time, $17 \mathrm{~s}$; and FOV, $400 \times 338 \mathrm{~mm} 5.5 / 2.1$; for dynamic contrast-enhanced MRI, a fix delay of $25 \mathrm{~s}$ was used for arterial phase images. Arterial and portal venous phase sequences were started 25 and $70 \mathrm{~s}$ after administration of IV contrast. The third phase was obtained immediately after the portal venous phase imaging. Each phase was obtained during separate breathholds. Injection rate: $2 \mathrm{~mL} / \mathrm{s}$.

\section{Image evaluation}

Qualitative evaluation. Two radiologists (ST and CST with 5 and 3 years of experience in abdominal MRI for observers 2 and 3, respectively), who were blinded to imaging reports, clinical history, and pathologic results retrospectively and independently reviewed first T2-w images alone, and then T2-w images and DW-MRI and ADC maps combined. To avoid the recall bias, the observers reviewed the MR images for detection of metastases in two separate sessions, each 3 weeks apart. Apparent diffusion coefficient (ADC) maps were generated from DW-MR images with commercial diffusion-analysis software (Advantage
Windows version 4.2.3, GE Healthcare, Milwaukee, WI, USA and Philips ViewForum, Philips Healthcare, Best, The Netherlands). Both readers recorded all malignant lesions and noted the number of lesions, their size, and image numbers on a data worksheet for each patient in both sessions. A maximum number of 10 malignant lesions per patient were recorded. Criteria for diagnosis of metastases were focal mildly to moderate hyperintense signal on T2-w image, bright signal on DW-MRI at both b-values and dark signal on ADC maps compared to the rest of the liver parenchyma $(3,7)$.

Consensus review as reference standard. Reference standard for number and characterization of the focal liver lesions was obtained from consensus review of DWMRI, ADC maps, T2-w and contrast enhanced T1-w images, pathologic data, and follow-up imaging results by observers 1,2 , and 3 . Metastases were diagnosed on the basis of clinical history, biopsy, or surgery results (in 17 patients), available PET-CT results (in six patients), MRI findings (including enhancement characteristics) (in two patients), and interval progression on follow-up examination (in 21 patients). The number, size, and location of the reference standard metastatic lesions for each patient were noted on a second worksheet.

Quantitative analysis. During the consensus review, the three radiologists selected one representative metastasis $(>1 \mathrm{~cm}$ in diameter) in consensus, for each patient for quantitative analysis. Observer 2 calculated ADC values by placing a region of interest over the entire lesion, as seen on the image with the largest lesion size using commercially available dedicated workstations (Advantage Windows version 4.2.3, GE Healthcare, Milwaukee, WI and Philips ViewForum, Philips Healthcare, Netherlands).

Statistical analysis. For all statistical analyses, a software package (SPSS 15.0 for Windows; SPSS Inc., Chicago, IL, USA) was used. Lesion detection rate of each session was calculated for each group and each observer and compared using McNemar Test.

Mean ADC values of hypo- and hypervascular lesions were compared with an unpaired two-tailed students t-test with a significance level of 0.05 . Simple $\kappa-$ coefficient was used to assess inter-observer variability.

\section{Results}

\section{Reference standard}

A total of 153 hypervascular and 187 hypovascular metastases were recorded at consensus review. 
The mean number of the hyper- and hypovascular metastases per patient was 6.9 (range, 2-10) and 7.4 (range, 1-10), respectively. The mean size of hypervascular metastases was $1.30+/-0.78 \mathrm{~cm}$, the mean size of hypovascular metastases was $1.64+7-1.35$.

\section{Detection of metastases}

The detection rates of hyper- and hypovascular metastasis by both observers are summarized in Tables 1 and 2, respectively. The detection rate of hypervascular metastases significantly improved when DW-MRI were reviewed together with $\mathrm{T} 2-\mathrm{w}$ images compared to $\mathrm{T} 2-\mathrm{w}$ images alone, for both observers (Table 1) (Figs. 1 and 2).

Although, more hypovascular metastases were detected on the combined DW-MRI and T2-W session by both observers, detection rate was not significantly better than $\mathrm{T} 2-\mathrm{w}$ images alone (Table 2) (Fig. 3).

The inter-observer agreement was moderate to substantial (kappa: 0.45 for $\mathrm{T} 2 \mathrm{w}$ and 0.41 for $\mathrm{T} 2 \mathrm{w}+\mathrm{DW}$ MRI in the group with hypervascular metastases; kappa: 0.79 for $\mathrm{T} 2 \mathrm{w}$ and 0.57 for T2w + DW-MRI in the group with hypovascular metastases).

\section{$A D C$ measurements}

In 19 patients with hypervascular metastases and in all 25 patients with hypovascular metastases, ADC values were measured. Two patients with hypervascular metastases were excluded, because the metastatic lesions were $<1 \mathrm{~cm}$ in diameter.

The mean ADC value of hypervascular metastases was significantly lower than mean ADC value of hypovascular metastases $\left(1.23+/-0.31 \times 10^{-3} \mathrm{~mm}^{2} / \mathrm{s} \quad\right.$ vs. $\left.1.49+/-0.19 \times 10^{-3} \mathrm{~mm}^{2} / \mathrm{s}\right)(P=0.001)($ Fig. 4$)$.

\section{Discussion}

Our results showed that the mean ADC value of hypervascular metastases was significantly lower than the mean ADC value of hypovascular metastases and additional review of DW-MR images provided significant improvement in detection of hypervascular metastases compared to the review of $\mathrm{T} 2-\mathrm{w}$ images alone.

With the advances in the hardware, sequence design and reconstruction, DW-MRI is increasingly used for the diagnosis of focal liver lesions. Benign hepatic lesions have generally higher ADC values compared to malignant lesions with variable degree of overlap (10). Different ADC cut-offs $\left(1.4-1.6 \times 10^{-3} \mathrm{~mm}^{2} / \mathrm{s}\right)$ have been described in the literature with a reported sensitivity of $74-100 \%$ and specificity of $77-100 \%$ for diagnosing malignant lesions (10-12). In these studies, mean ADC value of metastatic lesions varied between 0.94 and $1.50 \times 10^{-3} \mathrm{~mm}^{2} / \mathrm{s}(10-12)$. Technical factors such as the differences in the DW-MR image acquisition technique, different scanners and b-values utilized in these studies certainly play a role in these variations. However, in all of these studies, hypovascular and hypervascular metastatic lesions were grouped together

Table I. Detection rate of hypervascular metastases on two different review sessions by two observers.

\begin{tabular}{|c|c|c|c|c|}
\hline & \multicolumn{4}{|c|}{ Hypervascular metastases } \\
\hline & \multicolumn{2}{|l|}{ Observer I } & \multicolumn{2}{|l|}{ Observer 2} \\
\hline & T2-w & T2-w + DW-MRI & T2-w & T2-w + DW-MRI \\
\hline Detection rate & 129/I53 (84.3\%) & I48/I53 (96.7\%) & $113 / 153$ (73.8\%) & $125 / 153$ (8I.9\%) \\
\hline$P$ values & 0.002 & & 0.004 & \\
\hline
\end{tabular}

Table 2. Detection rate of hypovascular metastases on two different review sessions by two observers.

\begin{tabular}{|c|c|c|c|c|}
\hline & \multicolumn{4}{|c|}{ Hypovascular metastases } \\
\hline & \multicolumn{2}{|l|}{ Observer I } & \multicolumn{2}{|l|}{ Observer 2} \\
\hline & $\mathrm{T} 2-\mathrm{w}$ & T2-w + DW-MRI & $\mathrm{T} 2-\mathrm{w}$ & T2-w + DW-MRI \\
\hline Detection rate & I80/I87 (96.2\%) & 184/187 (98.4\%) & I76/187 (94.1\%) & $180 / 187(96.2 \%)$ \\
\hline$P$ values & 0.25 & & 0.125 & \\
\hline
\end{tabular}


and study groups were composed of different percentages of each metastatic subtype. We believe that inherent ADC difference between hypovascular and hypervascular metastasis described in our study may have also contributed to this variation.

In a study aiming to evaluate the role of DW-MRI in differentiation of hemangiomas from other hypervascular lesions, the mean ADC value of 64 hepatic metastatic lesions from neuroendocrine tumor was found to be $1.43 \times 10^{-3} \mathrm{~mm}^{2} / \mathrm{s}+/-0.39$ (13). Even though this was slightly higher than the mean ADC value of hypervascular metastasis in our study $\left(1.23 \times 10^{-3} \mathrm{~mm}^{2} / \mathrm{s}+/\right.$ -0.31 ), the difference is within the standard error of both measurements. Hypervascularity of tumors has been reported to reflect increased vascular density, increased mean vessel diameter, and greater vascular wall permeability $(14,15)$. Hypervascularity of liver lesions can also result from desmoplastic reaction, inflammatory cell infiltrates, and increased release of the vascular endothelial growth factor $(16,17)$. All of these factors in addition to the abundance of small, round cells in the histopathology of neuroendocrine tumors can contribute to the low ADC value of hypervascular metastasis by increasing the cellular density and decreasing the likelihood of tumor necrosis. On the other hand, in a separate study, the mean ADC value of colorectal hepatic metastasis (prototype of hypovascular metastases) was substantially higher $\left(1.94 \times 10^{-3} \mathrm{~mm}^{2} / \mathrm{s}\right)$ than the ADC range of the metastatic lesions described in the literature (18).
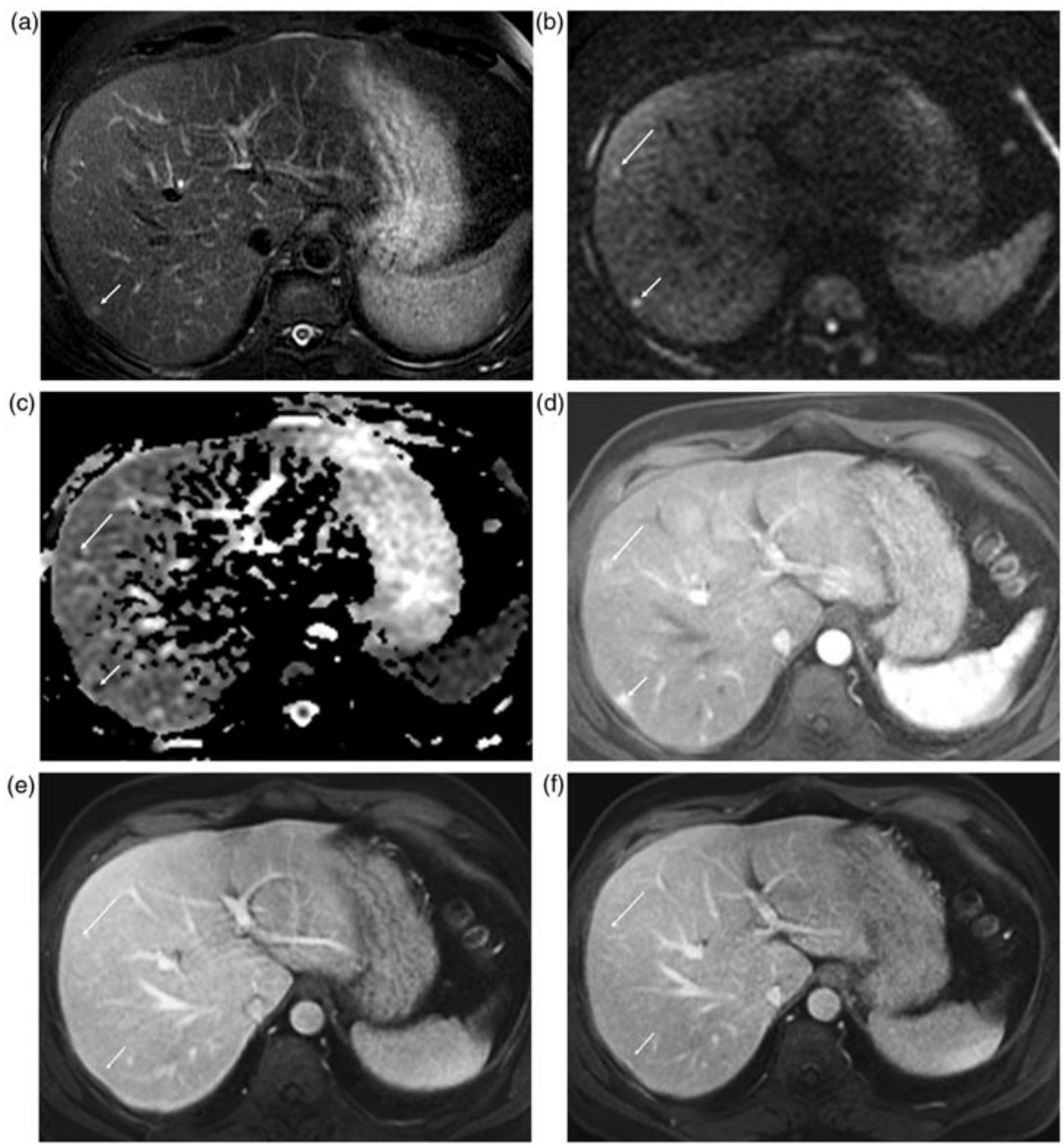

Fig. I. A 58-year-old man with hypervacular metastases of neurendocrine tumor. Axial T2-w fat-saturated fast spin-echo image (a) shows one slightly hyperintense lesion (short arrow) in the left lobe of the liver compatible with metastasis. In addition to this lesion, axial DW-MR image $b=800 \mathrm{~s} / \mathrm{mm}^{2}$ (b) reveals a second hepatic metastasis (long arrow) which cannot be detected on T2-w image. They show dark signal on ADC map (c) indicating restricted diffusion. These lesions were new from previous study and demonstrated homogenous arterial enhancement (arrows) on the contrast-enhanced TI-w gradient-echo image obtained at the arterial phase (d), whereas they show wash-out on portal venous (e) and venous images (f). 

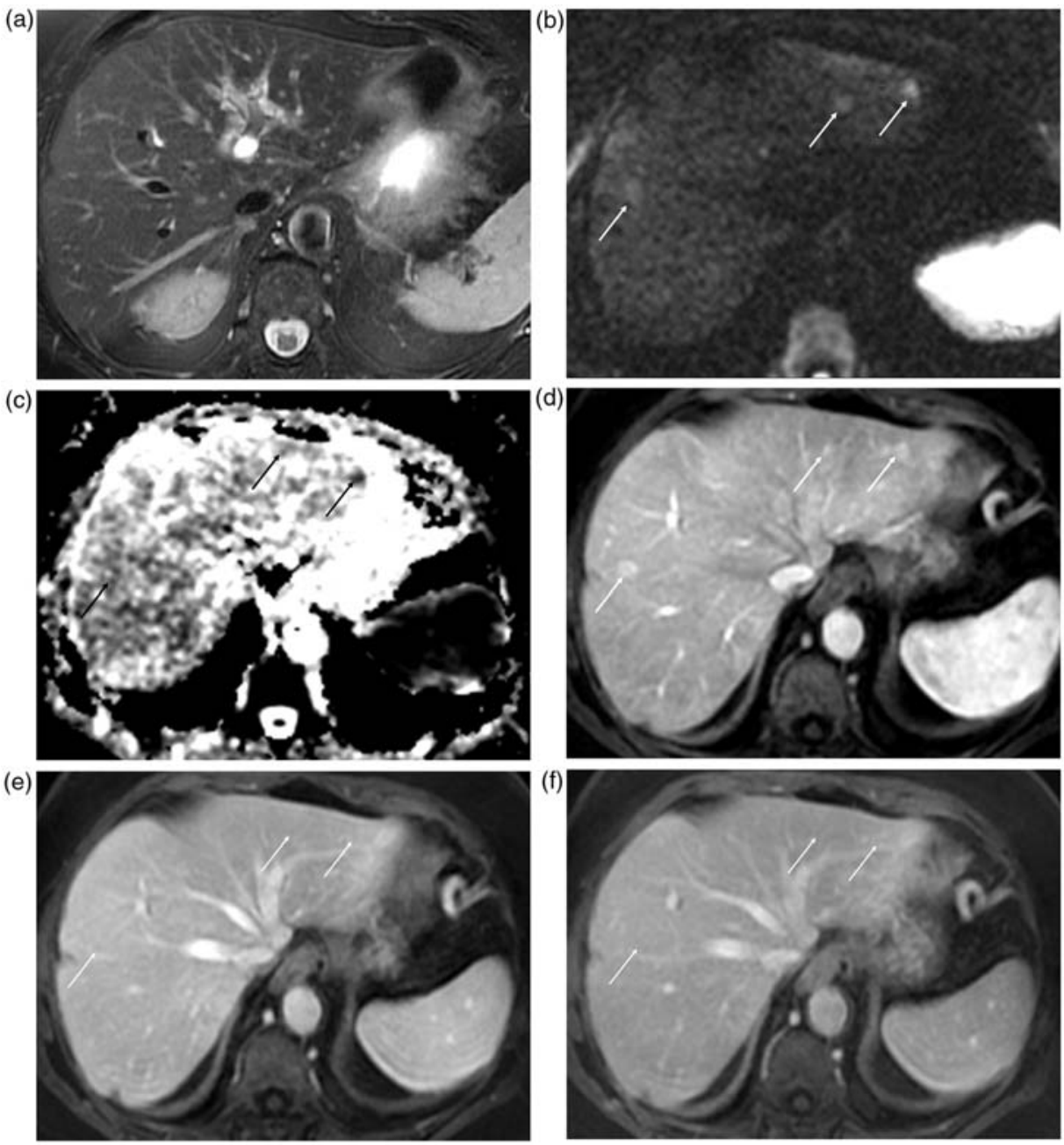

Fig. 2. A 70-year-old woman with hypervacular metastases of neurendocrine tumor. Axial T2-w fat-saturated fast spin-echo image (TR, 57I4.29; TE, 93.3) (a) does not show any hepatic metastases, whereas axial DW-MR image at $b=800 \mathrm{~s} / \mathrm{mm}^{2}(\mathrm{~b})$ at the same level demonstrate three hyperintense metastatic lesions (arrows) in the liver. They show dark signal on ADC map (c) indicating restricted diffusion. Contrast-enhanced TI-w gradient-echo image obtained at the arterial phase (d) shows the same three lesions (arrows) demonstrating homogenous arterial enhancement with wash-out on portal venous (e) and venous images (f), typical for hypervascular metastasis.

Substantial central necrosis can be commonly encountered in the hypovascular metastatic lesions causing increased ADC values (18).

Several studies comparing DW-MRI and T2-w imaging have shown improved detection of focal malignant lesions in the liver with the DW-MRI $(19,20)$. Bruegel et al. compared the value of respiratory-triggered DWMRI and five different T2-w turbo spin-echo sequences in detecting hepatic metastases (20). Sensitivity for the diagnosis of 118 liver metastases was significantly higher by using DW-MRI (0.88-0.91) than T2-W images (0.59-0.67) (20). Similar to this study, Parikh et al. found DW-MRI to be significantly better than T2-w imaging in detecting malignant focal liver lesions $(83.3 \%$ and $62.9 \%)$ (11). In our study, DW-MRI improved detection rate of only hypervascular metastasis compared to T2-w imaging. Dromain et al. compared four different MRI sequences for detection of hepatic metastases caused by neuroendocrine tumors and similar to our results, they reported a detection rate of $77.7 \%$ for $\mathrm{T} 2-\mathrm{w}$ images (21). In their study, hepatic arterial phase images detected the highest percentage of metastatic lesions $(89.1 \%)$, similar to our results of combined DW-MR and T2-w imaging session results ( $96.7 \%$ and $81.9 \%$ for observers 1 and 2, respectively) (21). These results suggest that DW-MRI can be a useful complimentary sequence to $\mathrm{T} 2-\mathrm{w}$ imaging for detection of hypervascular liver metastasis, especially when the patients have risk factors for gadolinium administration or an optimal arterial phase could not be obtained due to technical factors. Some of the small hypervascular metastatic lesions can only be seen at the 

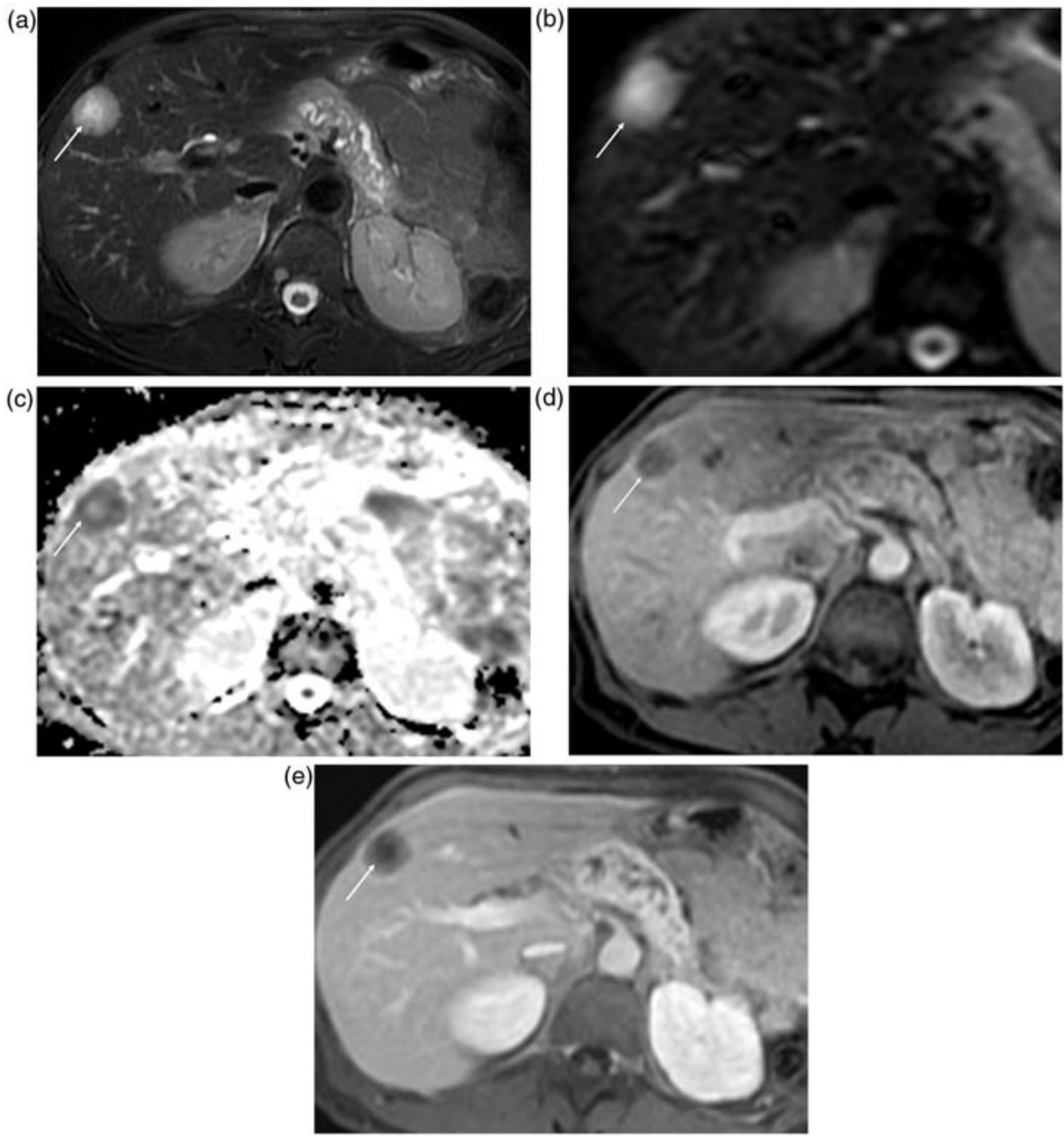

Fig. 3. A 65-year-old man with hypovascular metastases of lung cancer. Axial T2-w fat-saturated fast spin-echo image (TR, I0,000; TE, 95.I) (a) shows a slightly hyperintense metastasis (arrow) compared to the liver parenchyma, which demonstrates bright signal on transverse single-shot echo-planar DW image at $b=800 \mathrm{~s} / \mathrm{mm}^{2}$ (b). It shows dark signal (arrow) on ADC map (c) indicating restricted diffusion. Contrast-enhanced TI-w gradient-echo image on arterial phase (d) decreased enhancement compared to the normal liver parenchyma with peripheral rim enhancement of the metastasis in the portal venous phase (e).

arterial phase and become isointense on other dynamic phases (21). Since this enhancement characteristic can also be seen in focal nodular hyperplasia and adenomas, DW-MRI may potentially help differentiating metastasis from other lesions with benign etiology.

In our study, DW-MRI did not significantly improve the detection rate of hypovascular metastasis. On T2-w images, hypovascular metastases can have peripheral hyperintense rim representing live cancer cells surrounding central dark signal representing a combination of fibrosis and coagulation necrosis (22). Large hypovascular metastases may have central hyperintensity representing liquefactive necrosis $(2,3,18)$. These features can make hypovascular metastases more readily detectable on $\mathrm{T} 2-\mathrm{w}$ images and diminish the added value DW-MR images in their detection.
Our study has limitations due to its retrospective design. We included only patients with known liver metastases and this may have affected the sensitivity in the qualitative review and did not permit valid results about the specificity. Our patients were scanned on different MR platforms with inherent differences in parameters. These technical differences may have led to variation in the ADC measurements. However, a recent study demonstrated inter- and intra-system reproducibility of ADC measurements and the independence of ADC values on MR hardware, field strength, sequence parameters, and diffusion b-factors in a simple water phantom (23).

Additionally, pathologic diagnosis was not available for all patients and we did not differentiate between untreated and treated metastases. Furthermore information of previous therapies could not be obtained. 


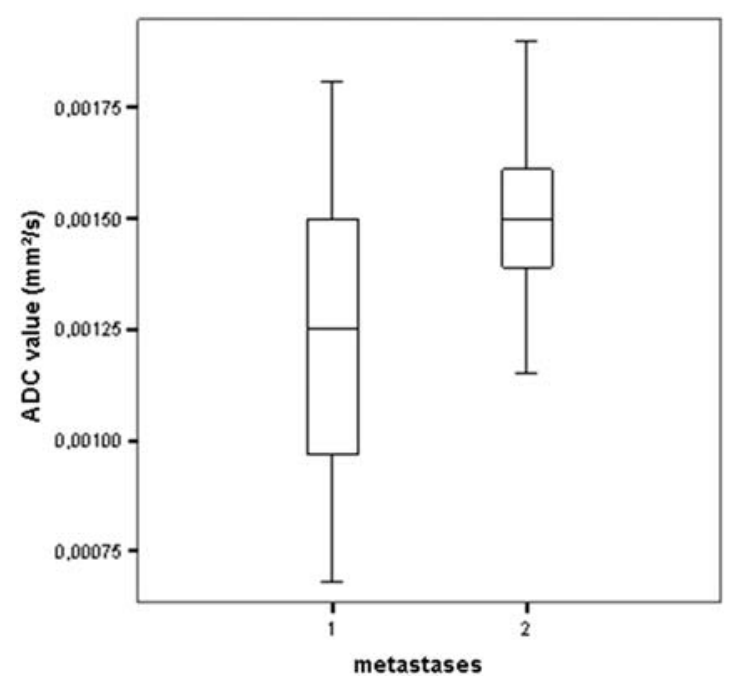

Fig. 4. Whisker-plot diagram of ADC values of hyper- and hypovascular metastases. Despite the substantial overlap between the ADC values of hypervascular (group I on the $\mathrm{x}$-axis) and hypovascular (group 2 in the $\mathrm{x}$-axis) metastatic lesions, mean $A D C$ value of hypervascular metastases $\left(1.23+/-0.31 \times 10^{-3} \mathrm{~mm}^{2} / \mathrm{s}\right)$ was significantly lower than mean ADC value of hypovascular metastases $(1.49+1$ $\left.-0.19 \times 10^{-3} \mathrm{~mm}^{2} / \mathrm{s}\right)(P=0.001)$.

Due to the retrospective nature of the study, only a single b-value was utilized. In addition cellularity, perfusion, fibrosis, tissue alterations due to therapy may influence the ADC measurements as well. Further prospective studies investigating the role of multiple b-values (especially smaller b-values allowing calculation of perfusion sensitive, fast ADC values) in the diagnosis of different types of metastases are warranted.

In conclusion, liver metastases are not a homogenous group of lesions with uniform DW-MRI features: hypervascular liver metastases demonstrate significantly lower ADC values compared to hypovascular metastatic lesions. DW-MRI improved detection of hypervascular liver metastases compared to $\mathrm{T} 2-\mathrm{w}$ images alone and can be a useful adjunct to T2-w images for their diagnosis, especially in patients with known risk factors for MR contrast agents.

\section{Funding}

This research received no specific grant from any funding agency in the public, commercial, or not-for-profit sectors.

\section{References}

1. Silva AC, Evans JM, McCullough AE, et al. MR imaging of hypervascular liver masses: a review of current techniques. Radiographics 2009;29:385-402.

2. Namasivayam S, Martin DR, Saini S. Imaging of liver metastases: MRI. Cancer Imaging 2007;7:2-9.
3. Namasivayam S, Salman K, Mittal $P$, et al. Hypervascular hepatic focal lesions: Spectrum of Imaging features. Curr Probl Diagn Radiol 2007;36:107-123.

4. Sandrasegeran K, Rajesh A, Rushing DA, et al. Gastrointestinal stromal tumors: CT and MRI findings. Eur Radiol 2005;15:1407-1414.

5. Elsayes KM, Narra VR, Yin Y, et al. Focal hepatic lesions: Diagnostic value of enhancement pattern approach with contrast-enhanced 3D gradient-echo MR imaging. Radiographics 2005;25:1299-1320.

6. Danet IM, Semelka RC, Leonardou P, et al. Spectrum of MRI appearances of untreated metastases of the liver. Am J Roentgenol 2003;181:809-817.

7. Ba-Ssalamah A, Uffmann M, Saini S, et al. Clinical value of liver-specific contrast agents: a tailored examination for a confident non-invasive diagnosis of focal liver lesions. Eur Radiol 2009;19:342-357.

8. Ward J. New MR techniques for the detection of liver metastases. Cancer Imaging 2006;6:33-42.

9. Shellock FG, Spinazzi A. MRI Safety update 2008: Part I, MRI contrast agents and nephrogenic systemic fibrosis. Am J Roentgenol 2008;191:1129-1139.

10. Taouli B, Koh DM. Diffusion-weighted MR imaging of the liver. Radiology 2010;254:47-66.

11. Parikh T, Drew SJ, Lee VS, et al. Focal liver lesion detection and characterization with diffusion-weighted MR-imaging: Comparison with standard breath-hold T2-weighted imaging. Radiology 2008;246:812-822.

12. Taouli B, Vilgrain V, Dumont E, et al. Evaluation of liver diffusion isotropy and characterization of focal hepatic lesions with two single-shot echo-planar MR imaging sequences: prospective study in 66 patients. Radiology 2003;226:71-78.

13. Vossen JA, Buijis M, Liapi E, et al. Receiver operating characteristic analysis of diffusion-weighted magnetic resonance imaging in differentiating hepatic hemangioma from other hypervascular liver lesions. J Comput Assist Tomogr 2008;32:750-756.

14. Mayr NA, Hawighorst H, Yuh WT, et al. MR microcirculation assessment in cervical cancer: correlations with histomorphological tumor markers and clinical outcome. J Magn Reson Imaging 1999;10:267-276.

15. Passe TJ, Bluemke DA, Siegelman SS. Tumor angiogenesis: tutorial on implications for imaging. Radiology 1997;203:593-600.

16. Semelka RC, Hussain SM, Marcos HB, et al. Perilesional enhancement of hepatic metastases: correlation between MR imaging and histopathologic findings - initial observations. Radiology 2000;215:89-94.

17. Neeman M, Provenzale JM, Dewhirst MW. Magnetic resonance imaging applications in the evaluation of tumor angiogenesis. Semin Radiat Oncol 2001;11:70-82.

18. Koh DM, Scurr E, Collins DJ, et al. Colorectal hepatic metastases: quantitative measurements using single-shot echo-planar diffusion-weighted MR imaging. Eur Radiol 2006;16:1898-1905.

19. Coenegrachts K, Delanote J, Ter Beek I, et al. Improved focal liver lesion detection: comparison of single-shot 
diffusion-weighted echoplanar and single-shot T2 weighted turbo spin echo techniques. $\mathrm{Br} \mathrm{J}$ Radiol 2007;80:524-531.

20. Bruegel M, Gaa J, Waldt S, et al. Diagnosis of hepatic metastasis: comparison of respiration-triggered diffusionweighted echo-planar MRI and five t2-weighted turbo spin-echo sequences. Am J Roentgenol 2008;191:1421-1429.

21. Dromain C, de Baere T, Baudin E, et al. MR imaging of hepatic metastases caused by neuroendocrine tumors: comparing four techniques. Am $\mathrm{J}$ Roentgenol 2003;180:121-128.

22. Haider MA, Farhadi FA, Milot L. Hepatic perfusion imaging: concepts and application. Magn Reson Imaging Clin N Am 2010;18:465-475.

23. Ivancevic MK, Meyer CR, Galban CJ, et al. Simple universal phantom for multi-center apparent diffusion coefficient (ADC) measurement. Proc Intl Soc Mag Reson Med 2009;17:4166. 